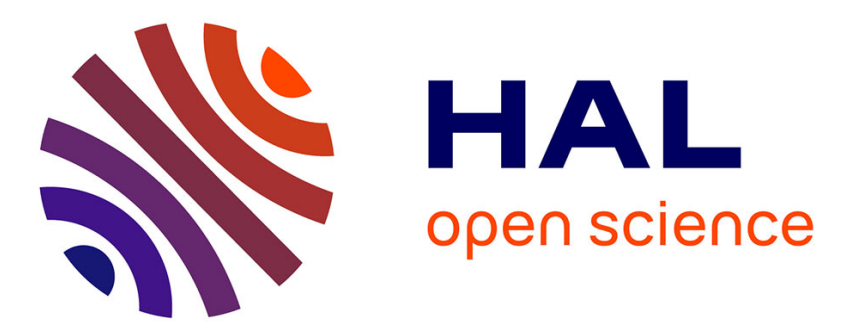

\title{
Influence of joint constraints on lower limb kinematics estimation from skin markers using global optimization
}

Sonia Duprey, Laurence Cheze, Raphaël Dumas

\section{To cite this version:}

Sonia Duprey, Laurence Cheze, Raphaël Dumas. Influence of joint constraints on lower limb kinematics estimation from skin markers using global optimization. Journal of Biomechanics, 2010, 43 (14), pp. 2858-2862. 10.1016/j.jbiomech.2010.06.010 . hal-00990413

\section{HAL Id: hal-00990413 \\ https://hal.science/hal-00990413}

Submitted on 13 May 2014

HAL is a multi-disciplinary open access archive for the deposit and dissemination of scientific research documents, whether they are published or not. The documents may come from teaching and research institutions in France or abroad, or from public or private research centers.
L'archive ouverte pluridisciplinaire HAL, est destinée au dépôt et à la diffusion de documents scientifiques de niveau recherche, publiés ou non, émanant des établissements d'enseignement et de recherche français ou étrangers, des laboratoires publics ou privés. 

using global optimization

3

4 Sonia Duprey ${ }^{\text {a*, }}$, Laurence Cheze ${ }^{\text {a }}$, Raphaël Dumas ${ }^{\text {a }}$ 5

9

$10 *$ Corresponding author:

11 Sonia Duprey, LBMC, INRETS, 25 av F Mitterrand, F-69675 Bron Cedex, France. Phone:

12 (+33) 478656882 ; Fax: (+33) 4721423 60; Email: sonia.duprey@univ-lyon1.fr 13

14 Word count: 1523 words 
Abstract (226 words)

2

3 In order to obtain the lower limb kinematics from skin-based markers, the soft tissue artefact

4 (STA) has to be compensated. Global optimization (GO) methods rely on a predefined

5 kinematic model and attempt to limit STA by minimizing the differences between model

6 predicted and skin-based marker positions. Thus, the reliability of GO methods depends

7 directly on the chosen model, whose influence is not well known yet.

8 This study develops a GO method that allows to easily implement different sets of joint

9 constraints in order to assess their influence on the lower limb kinematics during gait. The

10 segment definition was based on generalized coordinates giving only linear or quadratic joint

11 constraints. Seven sets of joint constraints were assessed, corresponding to different kinematic

12 models at the ankle, knee and hip: SSS, USS, PSS, SHS, SPS, UHS and PPS (where S, U and

$13 \mathrm{H}$ stand for spherical, universal and hinge joint and $\mathrm{P}$ for parallel mechanism). GO was

14 applied to gait data from five healthy males.

15 Results showed that the lower limb kinematics, except hip kinematics, knee and ankle 16 flexion-extension, significantly depend on the chosen ankle and knee constraints. The knee 17 parallel mechanism generated some typical knee rotation patterns previously observed in 18 lower limb kinematic studies. Furthermore, only the parallel mechanisms produced joint 19 displacements.

20 Thus, GO using parallel mechanism seems promising. It also offers some perspectives of 21 subject-specific joint constraints. 
3 To obtain an accurate skeleton kinematics from skin-based markers, the relative motion of

4 soft tissues with regards to the underlying bone (i.e. the soft tissue artefact, STA) has to be

5 compensated. Several methods minimizing STA have been developed (Leardini et al., 2005).

6 Some methods address each segment separately by computing the optimal bone pose from a

7 marker cluster (Söderkvist and Wedin, 1993; Challis, 1995, Cheze et al., 1995), while global

8 optimization (GO) methods address the entire limb, or full body, by minimizing distances

9 between measured and model-determined marker positions ( $\mathrm{Lu}$ and O'Connor, 1999). GO

10 methods rely on the determination of a predefined kinematic model with specific joint

11 constraints. Therefore, GO results directly depend on the constraint choices.

12 Spherical joints have been classically applied (Lu and O'Connor, 1999; Charlton et al., 2004).

13 Alternatively, models including universal and hinge joints at the ankle and knee were

14 proposed (Reinbolt et al., 2005; Andersen et al., 2009c). Moreover, degree-of-freedom coupling curves were included as knee joint constraints in a registration technique (Sholukha et al. 2006) providing reliable results in terms of joint displacements. However the coupling curves were dependent on the chosen segment axes and Euler angle sequence.

Coupled degrees-of-freedom can also be modelled by spatial parallel mechanisms (Feikes et al., 2003; Di Gregorio et al., 2007) that directly take into account anatomical structures (i.e. articular surfaces as sphere-on-plane contacts and ligaments as constant lengths). The corresponding joint constraints have not been included in GO methods so far.

The aim of the current study is to develop a GO method that allows to easily implement different sets of joint constraints, in order to assess their influence on the lower limb kinematics during gait. Different sets of joint constraints were evaluated, corresponding to 
1 different kinematic models at the ankle, knee and hip joints: SSS, USS, PSS, SHS, SPS, UHS

2 and PPS (where S, U and $\mathrm{H}$ stand for spherical, universal and hinge joint and P for parallel 3 mechanism).

6 Global optimization methods (see appendices $A, B, C$ for more details)

Parameter set

9 GO was performed using generalized coordinates (Dumas and Cheze, 2007) consisting, for each segment $i$, of two position vectors (the proximal $P_{i}$ and distal $D_{i}$ endpoints) and two 11 unitary direction vectors $\left(\mathbf{u}_{i}\right.$ and $\left.\mathbf{w}_{i}\right)$ :

13 with $i=1,2,3,4$ for the foot, shank, thigh, and pelvis, respectively.

These parameters were designed to stand for non-orthogonal directions: inertial (for inverse dynamics purpose), anatomical (i.e., from joint centre to joint centre) and functional (i.e., mean axis of rotation). Particularly, $\mathbf{r}_{D 2}$ and $\mathbf{r}_{P 2}$ are the ankle and knee joint centres while $\mathbf{w}_{2}$ and $\mathbf{w}_{3}$ are the ankle and knee flexion-extension axes. In addition, any position $\mathbf{r}$ of a point (marker or virtual) and any direction $\mathbf{n}$ embedded in the segment $i$ can be straightforwardly deduced from $\mathbf{Q}_{i}$ through a constant interpolation matrix $\mathbf{N}_{i}$ (Garcia de Jalon et al., 1986; Dumas and Cheze, 2007). As 12 parameters represent the 6 degrees of freedom of the segment, rigid body constraints have to be considered in addition to the joint constraints. 
3 The objective function to minimize is the sum of the square distances between measured and

4 model-determined marker positions:

$5 \quad f=\sum_{i=1}^{4} \sum_{j=1}^{m_{i}}\left(\mathbf{r}_{M_{i}^{j}}-\mathbf{N}_{i}^{M_{i}^{j}} \mathbf{Q}_{i}\right)^{2}$

6 with $M_{i}^{j}$ the $j^{\text {th }}$ marker (out of $m_{i}$ ) embedded in segment $i, \mathbf{r}_{M_{i}^{j}}$ its measured position and $\mathbf{N}_{i}^{M_{i}^{j}}$

7 the corresponding interpolation matrix.

8

9

10

Joint constraints

11

12 For the spherical model, the joint constraints at the ankle, knee and hip are:

$13 \quad \mathbf{r}_{D_{i+1}}-\mathbf{r}_{P_{i}}=0(i=1,2)$ and $\mathbf{N}_{4}^{V_{4}^{1}} \mathbf{Q}_{4}-\mathbf{r}_{P_{3}}=0$

14 with $V_{4}^{1}$ a virtual point (i.e., hip joint centre) embedded in the pelvis segment and $\mathbf{N}_{4}^{V_{4}^{1}}$ the 15 corresponding interpolation matrix.

16

17 For the universal joint at the ankle, the joint constraints are:

$18\left\{\begin{array}{c}\mathbf{r}_{D_{2}}-\mathbf{r}_{P_{1}}=0 \\ \mathbf{w}_{2} \bullet \mathbf{N}_{1}^{\mathrm{n}_{1}^{1}} \mathbf{Q}_{1}-\cos \theta_{A}=0\end{array}\right.$,

19 with $\theta_{A}$ the angle defining the relative orientation of the two functional joint axes, $\mathbf{n}_{1}^{1}$ an axis

20 (i.e., subtalar) embedded in the foot segment and $\mathbf{N}_{1}^{\mathbf{n}_{1}^{1}}$ the corresponding interpolation matrix.

22 For the hinge model at the knee, the joint constraints are: 
$1\left\{\begin{array}{c}\mathbf{r}_{D_{3}}-\mathbf{r}_{P_{2}}=0 \\ \mathbf{w}_{3} \bullet\left(\mathbf{r}_{P_{2}}-\mathbf{r}_{D_{2}}\right)-L_{2} \cos \theta_{K}^{1}=0, \\ \mathbf{w}_{3} \bullet \mathbf{u}_{2}-\cos \theta_{K}^{2}=0\end{array}\right.$

2 with $\theta_{K}^{1}$ and $\theta_{K}^{2}$ two angles defining the orientation of the joint axes and $L_{2}$ the shank

3 segment length.

4

5 For the parallel mechanism at the ankle (Di Gregorio et al., 2007), the joint constraints are:

$6 \quad\left\{\begin{array}{c}\left(\mathbf{N}_{1}^{V_{1}^{1}} \mathbf{Q}_{1}-\mathbf{N}_{2}^{V_{2}^{1}} \mathbf{Q}_{2}\right) \cdot \mathbf{N}_{2}^{\mathbf{n}_{2}^{1}} \mathbf{Q}_{2}-d_{A}^{1}=0 \\ \left(\mathbf{N}_{1}^{V_{1}^{2}} \mathbf{Q}_{1}-\mathbf{N}_{2}^{V_{2}^{2}} \mathbf{Q}_{2}\right) \cdot \mathbf{N}_{2}^{\mathbf{n}_{2}^{2}} \mathbf{Q}_{2}-d_{A}^{2}=0 \\ \left(\mathbf{N}_{2}^{V_{2}^{3}} \mathbf{Q}_{2}-\mathbf{N}_{1}^{V_{1}^{3}} \mathbf{Q}_{1}\right)^{2}-\left(d_{A}^{3}\right)^{2}=0 \\ \left(\mathbf{N}_{2}^{V_{2}^{4}} \mathbf{Q}_{2}-\mathbf{N}_{1}^{V_{1}^{4}} \mathbf{Q}_{1}\right)^{2}-\left(d_{A}^{4}\right)^{2}=0 \\ \left(\mathbf{N}_{2}^{V_{2}^{5}} \mathbf{Q}_{2}-\mathbf{N}_{1}^{V_{1}^{5}} \mathbf{Q}_{1}\right) \bullet \mathbf{N}_{1}^{\mathbf{n}_{1}^{2}} \mathbf{Q}_{1}-d_{A}^{5}=0\end{array}\right.$

7 These constraints represent the tibia/talus sphere-on-plane medial and lateral contacts (sphere

8 centres $V_{1}^{1}, V_{1}^{2}$ and radii $d_{A}^{1}, d_{A}^{2}$, contact plane points $V_{2}^{1}, V_{2}^{2}$ and normals $\mathbf{n}_{2}^{1}, \mathbf{n}_{2}^{2}$ ), the

9 calcaneum-tibia, calcaneum-fibula ligaments (origins $V_{2}^{3}, V_{2}^{4}$ insertions $V_{1}^{3}, V_{1}^{4}$ and lengths $\left.d_{A}^{3}, d_{A}^{4}\right)$ and the fibula/talus sphere-on-plane contact (centre $V_{2}^{5}$ and radius $d_{A}^{5}$, contact plane

11 point $V_{1}^{5}$ and normal $\mathbf{n}_{1}^{2}$ ).

12

13 For the parallel mechanism at the knee (Feikes et al., 2003), the joint constraints are:

$14\left\{\begin{array}{c}\left(\mathbf{N}_{3}^{V_{3}^{1}} \mathbf{Q}_{3}-\mathbf{N}_{2}^{V_{2}^{6}} \mathbf{Q}_{2}\right) \cdot \mathbf{N}_{2}^{\mathbf{n}_{2}^{3}} \mathbf{Q}_{2}-d_{K}^{1}=0 \\ \left(\mathbf{N}_{3}^{V_{3}^{2}} \mathbf{Q}_{3}-\mathbf{N}_{2}^{V_{2}^{7}} \mathbf{Q}_{2}\right) \cdot \mathbf{N}_{2}^{\mathbf{n}_{2}^{4}} \mathbf{Q}_{2}-d_{K}^{2}=0 \\ \left(\mathbf{N}_{3}^{V_{3}^{3}} \mathbf{Q}_{3}-\mathbf{N}_{2}^{V_{2}^{8}} \mathbf{Q}_{2}\right)^{2}-\left(d_{K}^{3}\right)^{2}=0 \\ \left(\mathbf{N}_{3}^{V_{3}^{4}} \mathbf{Q}_{3}-\mathbf{N}_{2}^{V_{2}^{9}} \mathbf{Q}_{2}\right)^{2}-\left(d_{K}^{4}\right)^{2}=0 \\ \left(\mathbf{N}_{3}^{V_{3}^{5}} \mathbf{Q}_{3}-\mathbf{N}_{2}^{V_{2}^{10}} \mathbf{Q}_{2}\right)^{2}-\left(d_{K}^{5}\right)^{2}=0\end{array}\right.$ 
1 These constraints represent the femur/tibia sphere-on-plane medial and lateral contacts

2 (sphere centres $V_{3}^{1}, V_{3}^{2}$ and radii $d_{K}^{1}, d_{K}^{2}$, contact plane points $V_{2}^{6}, V_{2}^{7}$ and normals $\mathbf{n}_{2}^{3}, \mathbf{n}_{2}^{4}$ ) and

3 the anterior cruciate, posterior cruciate and medial collateral ligaments (origins $V_{3}^{3}, V_{3}^{4}, V_{3}^{5}$

4 insertions $V_{2}^{8}, V_{2}^{9}, V_{2}^{10}$ and lengths $\left.d_{K}^{3}, d_{K}^{4}, d_{K}^{5}\right)$.

Model construction and initial guess

8 The construction of the lower limb kinematic models corresponds to the determination of the

9 constants (i.e. interpolation matrices $\mathbf{N}$, angles $\theta$, lengths $L$ and distances $d$ ) from static calibration, functional methods and literature data (Di Gregorio et al., 2007; Feikes et al., 11 2003).

In the optimization process, the initial values of the parameters are obtained by constructing at

13 each frame the position vectors $\mathbf{r}_{P i}$ and $\mathbf{r}_{D i}$ and direction vectors $\mathbf{u}_{i}$ and $\mathbf{w}_{i}$ from the skin-based markers (Dumas and Cheze, 2007; see also Appendix A).

Application

Five healthy male subjects (age: $28.8 \pm 4.8$ years; height: $1.74 \pm 0.09 \mathrm{~m}$; mass: $76.5 \pm 13.5 \mathrm{~kg}$ ) participated in this study. The trajectories of 32 skin-based markers on the right lower limb were recorded at $100 \mathrm{~Hz}$. The parameters $\mathbf{Q}_{i}$ were obtained by minimization of the objective function $f$ under constraints (i.e., rigid body and different sets of kinematic constraints) with

23 the use of the «fmincon» Matlab function (Mathworks, USA). Then, the classical segment coordinate systems were deduced from the $\mathbf{Q}_{i}$ parameters (Dumas and Cheze, 2007) and the 
1 joint angles and displacements were computed (Wu et al. 2002; see also Appendix C). RMS

2 differences were computed for each curve and averaged for the 5 subjects.

\section{Results}

6 Constraint influence

7 The hip kinematics was not much affected by ankle and knee constraint variations (Fig. 1,

8 Table 1): all models provided similar patterns and amplitudes for the hip flexion-extension

9 and abduction-adduction, and the dispersion of the internal-external rotations remained low.

10 The knee flexion-extension curves did not vary across models: a single pattern could be 11 observed and the dispersion was low. The patterns of the abduction-adduction, internal12 external rotation and displacement curves appeared to depend on the applied knee constraint 13 (Fig. 2), but not on the ankle constraint. However, ankle constraints caused a dispersion of the 14 internal-external rotations (Table 1).

15 The ankle flexion-extension curves showed a similar pattern for all models with a slight 16 dispersion (Fig. 3). The ankle abduction-adduction, internal-external rotation and 17 displacement curves varied whenever the ankle or knee constraint was modified (Tables 1 and 2), except for the PPS and PSS models which provided similar rotation patterns and, for some subjects, similar displacement patterns.

Comparison with in-vivo (intra-cortical pins) data from the literature

22 The hip kinematics reproduced typical patterns (the authors were not aware of any hip in-vivo data).

24 The knee flexion-extension biphasic pattern (Lafortune et al., 1992; Benoit et al., 2006; 25 Andersen et al., 2009a) was obtained for all models. Abduction-adduction of limited 
1 amplitude and internal rotation occurring twice during the stance phase (at heel strike and toe-

2 off) was found when the model included a knee parallel mechanism. Furthermore, only the 3 knee parallel mechanism could reproduce the femoral rollback.

4 The ankle flexion-extension curves reproduced the typical 2-peak pattern (Reinschmidt et al., 1996; 1997). All models roughly produced an initial abduction followed by a slight adduction

6 during stance and internal-external rotations of limited amplitude, as observed in in-vivo 7 studies (Reinschmidt et al., 1996; 1997). Besides, only the parallel mechanisms produced 8 joint displacements.

9

10

\section{Discussion and conclusions}

GO with joint constraints is one of the methods developed for minimizing STA. Its reliability is under controversy (Stagni et al., 2009; Andersen et al., 2009a). Nevertheless, defining a kinematic model is becoming usual in gait analysis (e.g., inverse-forward dynamics, musculoskeletal models) and the constraint choice is essential.

In this study, a GO method was developed to implement different sets of joint constraints. The segment definition, based on generalized coordinates (Dumas and Cheze, 2007), allowed to readily implement complex constraints. Besides, the classical segment and joint coordinate systems could easily be deduced.

Results showed that the lower limb kinematics, except hip kinematics, knee and ankle flexion-extension, significantly depend on the chosen set of constraints. An appropriate choice, such as the parallel mechanisms, seemed to provide physiologic patterns (i.e., limited abduction-adduction at the knee and femoral rollback). Furthermore, these mechanisms offer the possibility of matching model geometry with MRI data (e.g., by customizing the femur 
1 condyles centre and radius) and of adapting the model to pathologies (e.g., by suppressing a

2 ligament constraint). Thus, these mechanisms might be able to provide efficient subject3 specific models for clinical applications.

4 A major limitation of this study is the lack of in-vivo data. The ability to reproduce inter5 subject variability, which is an important criterion in the constraint choice, could not be 6 assessed and subject-by-subject validation could not be performed. Thus, further studies 7 enabling subject-by-subject comparisons would offer interesting perspectives.

\section{References}

Andersen, M.S., Benoit, D.L., Damsgaard, M., Ramsey, D.K., Rasmussen, J., 2009a. Do kinematic models reduce the effects of soft tissue artefacts in skin marker-based motion analysis? An in vivo study of knee kinematics. J Biomech, In Press, (available online doi:10.1016/j.jbiomech.2009.08.034).

16

Andersen, M. S., Damsgaard, M., Macwilliams, B., Rasmussen, J., 2009b. A computationally efficient optimisation-based method for parameter identification of kinematically determinate and over-determinate biomechanical systems. Comput Methods Biomech Biomed Engin, In 20 Press.

Andersen, M. S., Damsgaard, M., Rasmussen, J., 2009c. Kinematic analysis of over- 
1 Benoit, D.L., Ramsey, D.K., Lamontagne, M., Xu, L., Wretenberg, P., Renström P., 2006.

2 Effect of skin movement artifact on knee kinematics during gait and cutting motions 3 measured in vivo. Gait \& Posture 24, 152-164.

4

5 Challis, J.H., 1995. A procedure for determining rigid body transformation parameters. J 6 Biomech 28(6), 733-7.

7

8 Charlton, I.W., Tate, P., Smyth, P., and Roren, L., 2004. Repeatability of an optimised lower 9 body model. Gait \& Posture 20, 213-221.

10 analyses based on videa system data. J Biomech 28(7), 879-884.

13

14 modelling the mobility of the human knee joint. J Biomech 36(1), 125-129.

Di Gregorio, R., Parenti-Castelli, V., O’Connor, J.J., and Leardini, A., 2007. Mathematical models of passive motion at the human ankle joint by equivalent spatial parallel mechanisms. Med Bio Eng Comput 45, 305-313.

Dumas, R., and Cheze, L., 2007. 3D inverse dynamics in non-orthonormal segment coordinate system. Med Bio Eng Comput 45, 315-322.

Feikes, J.D., O’Connor, J.J., and Zavatsky, A.B., 2003. A constraint-based approach to

Cheze, L., Fregly B.J., Dimnet, J., 1995. A solidification procedure to facilitate kinematic analyses based on videa system data. J Biomech 28(7), 879-884. (1) 
1 Franci, R., Parenti-Castelli, V., Belvedere, C., Leardini, A. et al., 2009. A new one-DOF fully

2 parallel mechanism for modeling passive motion at the human tibiotalar joint. $\mathrm{J}$ Biomech 3 42(10), 1403-1408.

4

5 Garcia de Jalon, J., Unda, J., and Avello, A., 1986. Natural coordinates for the computer 6 analysis of multibody systems. Computer methods in applied mechanics and engineering, 56, $7 \quad 309-327$.

9 Lafortune, M.A., Cavanagh, P.R., Sommer III, H.J., Kalenak, A., 1992. Three-dimensional kinematics of the human knee during walking. J Biomech 25(4), 347-357.

Leardini, A., Chiari, L., Della Croce, U., and Cappozzo, A., 2005. Human movement analysis using stereophotogrammetry. Part 3: Soft tissue artifact assessment and compensation. Gait \& Posture 21, 212-225.

Lu, T.-W., and O'Connor, J.J., 1999. Bone position estimation from skin marker co-ordinates using global optimisation with joint constraints. J Biomech 32(2), 129-134.

Reinbolt, J.A., Schutte, J.F., Fregly, B.J., Koh, B.I., Haftkab, R.T., George, A.D. and Mitchell, K.H., 2005. Determination of patient-specific multi-joint kinematic models through 21 two-level optimization. J Biomech 38(3), 621-626.

23 Reinschmidt, C., 1996. Three-dimensional tibiocalcaneal and tibiofemoral kinematics during 24 human locomotion - measured with external and bone markers. PhD report, University of 25 Calgary, Alberta, Canada. 
2 Reinschmidt, C., van den Bogert, A. J., Murphy, N., Lundberg, A., Nigg B.M., 1997.

3 Tibiocalcaneal motion during running, measured with external and bone markers. Clinical

4 Biomech 12(1), 8-16.

5

6 Sholukha, V., Leardini, A., Salvia, P., Rooze, M., Van Sint Jan, S., 2006. Double-step

7 registration of in vivo stereophotogrammetry with both in vitro 6-DOFs electrogoniometry 8 and CT medical imaging. J Biomech 39(11), 2087-2095.

9

Söderkvist, I., and Wedin, P.A., 1993. Determining the movements of the skeleton using well11 configured markers. J Biomech 26(12), 1473-1477.

12 Stagni, R., Fantozzi, S., Cappello, A., 2009. Double calibration vs. Global optimisation: Performance and effectiveness for clinical application. Gait \& Posture 29, 119-122.

14

Wu, G., van der Helm, F.C.T., Veeger, H.E.J., Makhsous, M., Van Roy, P., Anglin, C., 16 Nagels, J., Karduna, A.R., McQuade, K., Wang, X., Werner, F.W., Buccholz, B., 2002. ISB 17 recommendation on definitions of joint coordinate system of various joints for the reporting of human joint motion—part I: ankle, hip, and spine. J Biomech 38(5), 981-992. 